\title{
BMJ Open PositivMasc: masculinities and violence against women among young people. Identifying discourses and developing strategies for change, a mixed-method study protocol
}

\author{
M Salazar (D , ${ }^{1}$ N Daoud, ${ }^{2}$ Claire Edwards, ${ }^{3,4}$ Margaret Scanlon, ${ }^{4}$ C Vives-Cases ${ }^{5,6}$
}

To cite: Salazar M, Daoud N, Edwards C, et al. PositivMasc: masculinities and violence against women among young people. Identifying discourses and developing strategies for change, a mixed-method study protocol. BMJ Open 2020;10:e038797. doi:10.1136/ bmjopen-2020-038797

- Prepublication history for this paper is available online. To view these files, please visit the journal online (http://dx.doi. org/10.1136/bmjopen-2020038797).

Received 24 March 2020 Revised 20 August 2020 Accepted 27 August 2020

Check for updates

(C) Author(s) (or their employer(s)) 2020. Re-use permitted under CC BY-NC. No commercial re-use. See rights and permissions. Published by BMJ.

For numbered affiliations see end of article.

Correspondence to

Dr M Salazar;

mariano.salazar@ki.se

\section{ABSTRACT}

Introduction Despite public policies and legislative changes aiming to curtail men's violence against women (VAW) around the world, women continue to be exposed to VAW throughout their life. One in three women in Europe has reported physical or sexual abuse. Men who display unequitable masculinities are more likely to be perpetrators. VAW is increasingly appearing at younger ages. The aims of the project are fourfold: (1) to explore and position the discourses that young people (men and women, 18-24 years) in Sweden, Spain, Ireland and Israel use in their understanding of masculinities, (2) to explore how these discourses influence young people's attitudes, behaviours and responses to VAW, (3) to explore individual and societal factors supporting and promoting anti-VAW masculinities discourses and (4) to develop actions and guidelines to support and promote anti-VAW masculinities in these settings.

Methods and analysis A participatory explorative mixedmethod study will be used. In Phase 1, qualitative methods will be used to identify the discourses that young people and stakeholders use to conceptualise masculinities, VAW and the actions that are needed to support and promote antiviolence masculinities. In Phase 2, concept mapping will be used to quantify the coherence, relative importance and perceived relationship between the different actions to support and promote anti-VAW masculinities. Phase 3 is a knowledge creation and translation phase, based on findings from Phases 1 and 2, where actions and guidelines to promote and support anti-VAW masculinities will be developed.

Ethics and dissemination Ethical clearance has been obtained from ethics review boards in each country. Results will be disseminated through peer-reviewed publications, presentations at international conferences, policy briefs, social media and through the project online hub. With its multicountry approach, our project results seek to inform policies and interventions aimed at promoting discourses which challenge hegemonic masculinities.

\section{INTRODUCTION}

The United Nations defines violence against women (VAW) as 'any act of gender-based
Strengths and limitations of this study

- Our mixed-method exploratory (MM) design allow us to combine the strengths of both qualitative and quantitative research to obtain a holistic picture of antiviolence masculinities in four countries.

- The participatory design of this project engages young people and community stakeholders.

- The methodology is based around a series of interconnected phases, each building on the previous one; qualitative data are used as a basic for the quantitative concept mapping study.

- The exploratory longitudinal MM design is time consuming and expensive compared with crosssectional studies.

- The statistical generalisation of the quantitative findings is limited.

violence that results in, or is likely to result in, physical, sexual or psychological harm or suffering to women, including threats of such acts, coercion or arbitrary deprivation of liberty, whether occurring in public or in private life'. ${ }^{1}$ Despite public policies and legislative changes aiming to curtail men's VAW around the world, women continue to be exposed to violence throughout their life course.

According to the 2015 European Union survey on $\mathrm{VAW},{ }^{2}$ one in three women in Europe has reported physical or sexual abuse from the age of 15 years. ${ }^{2}$ Rates vary between countries; the lifetime prevalence of physical/ sexual violence ranges from $46 \%$ in Sweden to $22 \%$ and $26 \%$ in Spain and Ireland, respectively. Meanwhile, in Israel, a recent nationwide study that included a stratified sample of perinatal women found that close to $40 \%$ of women attending maternal and child health services had been exposed to different forms of intimate partner violence (IPV) at some 
point in their lives, with the prevalence of IPV higher among Arab women $(67 \%)$ than among Jewish women $(27 \%){ }^{3}$

\section{Masculinities and VAW: what do we know?}

Connell ${ }^{4}$ proposes that there are different types of masculinities, which are related to each other in diverse ways (relations of hegemony, subordination, complicity and marginalisation). Among those, hegemonic masculinities are the constellations of actions that protect and reinforce the dominant position of men in a society. ${ }^{4}$ They represent the ideals of what it is to be a man in a given society. While they vary across settings, they often depict men as heterosexual, strong, as household heads, economic providers, entitled to sex and above all and dominant over women. ${ }^{4}$

Population-based studies in South Africa ${ }^{5}$ and multicountry studies in Latin America ${ }^{6}$ and Asia $^{7}$ have consistently found that men who display hegemonic masculinities are more likely to perpetrate different forms of VAW. A multicountry study in Asia found that men who reported adherence to traditional forms of masculinities (ie, low equality) were two times more likely to have committed sexual or physical abuse towards women (AIRR 2.22,95\% CI 1.28 to 3.84 ). ${ }^{7}$

However, masculinities can manifest themselves in many ways, and not always harmfully. In our previous qualitative studies in Latin America, for example, we found that young men enact different masculinities that express diverse discursive positions on gender equity and VAW. ${ }^{8-10}$ The discursive positions range from a challenging discourse that problematises gender inequality, men's lack of responsibility for VAW and sexual abuse and women's subordination to discourses that reject men's responsibility and curtail women's agency. Most promisingly, we have identified what we might refer to as anti-VAW masculinities existing among young men. These young men are more likely to reject risk behaviours traditionally associated with masculinity, to defend women's rights, to reject men's VAW and to actively be involved in actions preventing or curtailing VAW. ${ }^{8-10}$ These ideas have consequences for our understandings of masculinities in relation to VAW. These are ideas that we intend to explore further in this study, in European and Israeli contexts.

\section{Gender, power, masculinity and its relationship to VAW}

VAW does not occur in isolation as it is strongly influenced by gender and the way masculinities are enacted. Gender can be defined as the evolving, socially constructed and time-bound set of power, economic, emotional and symbolic relations by which men and women interact with each other. ${ }^{11}$ The concept of power is central in the study of VAW and gender. While there are many definitions, ${ }^{12}$ in this protocol power is defined as the ability to dominate others. ${ }^{12}$

Gender relations are often unequal, and the gender order is often viewed as a norm system in which men have a dominant position over women. This power is largely exercised by controlling (often through violence) women's lives and bodies. ${ }^{11}$ Therefore, it is clear that understanding masculinities is crucial if VAW is to be reduced.

Masculinities are constructed, reconstructed and sustained in multiple arenas in a society. For young people, these arenas include the educational system. ${ }^{13}$ From a symbolic perspective, the dichotomisation and conceptualisation of gender in terms of binary categories is reinforced by having separate restrooms, dividing boys and girls in sports activities, creating competition between genders and so on. Male pupils often establish hierarchies based on aggression, dominance, competition and sexuality that are reflections of the traditional gender order (ie, nerds vs the athletes, gay vs straights, etc). ${ }^{13}$ These traditional gender hierarchies between young men (and young women) are often tolerated if not supported.

However, gender relations are not static as they are constantly evolving. They are frequently challenged by men and women rejecting the gender order and its relationships of subordination and marginalisation. ${ }^{4}$ Howson $^{14}$ has proposed that protest masculinities and femininities exist, and are represented by men and women who challenge traditional gender relations. In Europe, Elliot has described a form of protest masculinity called caring masculinity. ${ }^{15}$ Caring masculinities reject men's domination over women and embrace positive values such as caring for others, interdependence and emotional expression.

Protest masculinities can also arise from men's involvement with groups challenging men's VAW. ${ }^{16}$ Developing anti-VAW masculinities is a process that takes time. ${ }^{8-10}$ It involves not only being non-violent oneself, but being agents of change by questioning and deterring VAW whenever it is enacted in a society. ${ }^{16}$ The outcomes of this process are influenced by the intensity of men's participation in these groups, the ideologies underpinning the group's work and societal factors supporting or hindering change. ${ }^{15}$

Flood $^{16}$ has highlighted that developing anti-VAW masculinities does not imply that men will also question the other privileges that patriarchy bestows to men (ie, greater decision-making power and authority, lack of involvement in household duties, etc). One example of this is benevolent sexism. ${ }^{17}$ Benevolent sexism is a paternalistic approach that views women as weak, vulnerable and without agency. This approach fosters men's protective stance over women; however, it also rationalises women's subordinated position in a society. ${ }^{17}$

\section{Rationale}

It is widely recognised that traditional hegemonic forms of masculinities must be challenged and addressed if any reduction on VAW is to be achieved. ${ }^{18}$ The European Commission Advisory Committee on Equal Opportunities for Women and Men has also highlighted the relevance 
of identifying and supporting new forms of masculinities in tackling gender inequality. ${ }^{19}$

In Europe, a significant amount of research has been conducted on hegemonic masculinity and its connection with violence towards other men, ${ }^{20-22}$ IPV/honour-related violence ${ }^{23}$ fatherhood, ill health and increased young men's risky sexual behaviours ${ }^{23}$ among others. These studies have provided important insights into the relationship between hegemonic masculinities, ill health and different types of violence. However, none have specifically focused on young people, on interrogating the existence of masculinities that specifically challenge VAW, and how these anti-VAW masculinities can be supported.

Our research focuses on young people since different forms of VAW, including IPV, are increasingly appearing at earlier ages. According to WHO data ${ }^{24}$ young men who are violent towards women have a higher risk of continuing being violent with their future partners. In addition, toxic partner relations can begin and endure if VAW is normalised at this age. Thus, VAW prevention should start early in life if sustained progress on preventing VAW is to be achieved. We believe that identifying young people's discursive positions on VAW, as well as the factors supporting and promoting anti-VAW masculinity discourses, are key steps in designing and implementing gender-sensitive, evidence-based policies aimed at reducing VAW. Through its methodology, participatory process and dissemination strategies, this research will seek to contribute to public and policy debate and actions around anti-VAW masculine identities in addressing VAW.

\section{Objectives}

The aim of this project is to explore the discourses that young people in Sweden, Spain, Ireland and Israel use in their understanding of masculinities and to explore how these discourses influence young people's attitudes, behaviours and responses to VAW. Furthermore, we will explore individual and societal factors supporting and promoting anti-VAW masculinity discourses within and across these countries and develop actions and guidelines to support and promote anti-VAW masculinities in these settings.

\section{METHODS AND ANALYSIS \\ Overall project design}

This research will be conducted in Sweden, Spain, Israel and Ireland. These countries were selected because the collaborating institutions are located there. We will conduct a participatory, mixed-method (MM) study using an exploratory sequential design. ${ }^{25}$ In the first phase, data will be gathered using semistructured interviews and focus groups discussions (FGDs) in order to identify the discourses that young people and stakeholders use to conceptualise masculinities and VAW, as well as actions needed to support and promote anti-VAW masculinities. In Phase 2, we will use concept mapping (CM) to quantify the coherence, relative importance and perceived relationship between the different actions to support and promote anti-VAW masculinities identified in Phase 1. Phase 3 is knowledge creation and translation phase where we will disseminate our findings and design guidelines, in conjunction with stakeholders and young people, to promote and support anti-VAW masculinities.

The project started on January 1st, 2019. Phase 1: The first 6 months of the project were focused on developing the project logistics and data collection instrument. Data collection for Phase 1 started on August 1st, 2019 and ended on February 29th, 2020. Phase 2: Planning for logistics and developing the data collection instrument started on March 1st, 2020 and ended on August 30th, 2020. Data collection for Phase 2 will be conducted from September 1st, 2020 to January 30th, 2021. Data analysis from both phases will be conducted from February 1st 2021 to August 30, 2021. Phase 3 started on September 1st, 2020 and will end on March 30th, 2022.

\section{Phase 1}

\section{Qualitative study with young men and women} Aim

(1) To explore and position the discourses that young people use in their understanding of masculinities, (2) to explore how these discourses influence young people's attitudes, behaviours and responses to VAW and (3) to explore individual and societal factors supporting and promoting anti-VAW masculinity discourses in the study settings.

\section{Participant selection}

Participants will be young people aged 18-24 years. Purposeful sampling ${ }^{26}$ of young people will be undertaken based on the primary criteria of age, gender and whether or not the young person is involved in activism or an organisation working to prevent VAW. Within the sample in each country, we will seek to obtain an equal representation of men and women, of different ages within the 18-24 age bracket, and also seek to capture representation based on diverse socioeconomic backgrounds and educational levels. Due to ethical reasons, we will not ask our participants for their sexual orientation or gender identity. However, young people are welcomed to participate regardless of their sexual orientation or gender identity.

The sampling strategy described above will allow us to compare the similarities and differences between different groups of young people based on age, gender and whether they are engaged or not in anti-VAW activism. Participants will be recruited through advertisements and flyers posted in educational institutions (high schools, universities, etc.), social media (Facebook, Instagram, etc.), non-governmental organisations (NGOs) and state institutions working with young people.

\section{Data collection}

In-depth interviews and FGDs will be used to gather the data. In-depth interviews will allow us to explore 
individual experiences and FGDs will allow us to identify community norms and values related to our research questions. A semistructured discussion guide with openended questions will be used to collect the data. This will be developed based on a literature review, our previous experience in the field and discussions with the countrylevel advisory groups of both stakeholders and young people established for the project.

The topics to be explored in the interviews and FGDs include perceptions about masculinities, femininities, different forms of VAW (emotional, physical, sexual, controlling behaviour and online violence) and suggestions about how to promote and support anti-VAW masculinities. Short vignettes describing everyday situations where VAW is enacted will be used to encourage discussion.

Data will be collected until saturation of the information is reached. Around 15-20 in-depth interviews will be conducted per country. Four FGDs, two with young people from the general population and two with young people affiliated with organisations working to prevent VAW, will be also conducted per country. FGDs will be stratified by gender. Non-binary and gender queer people will be welcomed to be part of the FGD they feel more comfortable with. Data will be audio recorded and transcribed verbatim.

\section{Analysis}

Situational analysis ${ }^{27}$ will be used to analyse the data. We have chosen this method because it allows us to identify how discourses on masculinity and VAW differ and overlap within and across settings. Discourses can be defined as 'a broadly discernible cluster of terms, descriptions and figures of speech often assembled around metaphors or vivid images' ${ }^{28}$ They are important because they reflect how people understand different topics and act on them.

Situational analysis starts by inductively coding the text and constantly comparing the data to group the codes into categories of meaning. The constant comparison will allow us to identify how the categories merge into discourses and what axes of variation (ie, responsibility for VAW) are present in the data. The data will be represented using a positional map articulating the different positions taken or not in the masculinity discourses around different axes of variation. ${ }^{27}$

\section{Qualitative study with stakeholders}

Aim

To identify individual and societal factors and strategies to support and promote anti-VAW masculinity discourses within and across the study settings.

\section{Participant selection and data collection}

Participants will be community (NGOs and youth organisations) and government stakeholders (ministries of social work, health, education, gender equality, etc) working in the prevention of VAW with young people. Participants will be identified through a combination of snowball sampling ${ }^{26}$ and a web search of institutions fitting the required profile.

In-depth interviews will be used to collect the data. A semistructured discussion guide will be developed based on a literature review and discussions with the national advisory groups. Topics to be explored include: (1) general information about the organisation (aims, target groups, activities, etc), (2) how VAW is conceptualised in their work and/or programmes and (3) suggestions for strategies to promote and support antiviolence masculinities in their setting. Around 10-15 in-depth interviews will be conducted in each country. Data will be audio recorded and transcribed verbatim.

Analysis

Qualitative content analysis will be used to analyse the data. ${ }^{29}$ The transcripts will be coded inductively looking at the manifest and latent content of the text. Codes will be categorised into a group of content that shares a commonality (categories). Later themes representing the overall meaning running through categories will be identified.

\section{Phase 2: CM}

Aim

To quantify the coherence, patterns of priorities and perceived relationship between the different actions for supporting and promoting anti-VAW masculinities identified in Phase 1.

CM is an MM approach that enables groups of actors to visualise their ideas around an issue of mutual interest and develop common frameworks through a structured participatory process. The steps are: (1) generation of ideas, (2) structuring of ideas through sorting and rating and (3) development of conceptual maps, and finally a collective interpretation of the maps. ${ }^{30}$

\section{Data collection and analysis}

Results of the interviews and FGDs identified in Phase 1 will be the starting point (brainstorming phase) for our CM study. The research team will then gather all the proposed actions for supporting and promoting anti-VAW masculinities and create a consolidated list containing between 50 and 100 individual statements or actions. ${ }^{30}$ A questionnaire containing the refined list will be sent by e-mail again to the study participants for sorting and rating. For the sorting, participants will be asked to group together actions in different groups based on their own judgments. For rating, the participants will be asked to rate each action in terms of both importance and current presence in their communities (a Likert Scale will be used).

GroupWisdom CM software (Concept System Incorporated, 2019) will be used to collect the data, and for analysis. The analysis will be conducted in different steps. Once all participants have sorted the strategies, multidimensional scaling (MDS) ${ }^{3031}$ will be applied to the sorting data. MDS assigns coordinates to each strategy locating 
them with a point in a bidimensional map to create a point map. The closer the points are to each other in the map, the more likely that the strategies represented by the points have been sorted together. Hierarchical cluster analysis is then performed to divide all strategies into clusters creating a cluster map. ${ }^{30}$ After this, the rating data are averaged for each strategy and cluster and displayed on top of the point and cluster maps, respectively. This is done to obtain a visual representation of the relative importance of the strategies within and between clusters. It also provides a visual representation of the relative importance of each cluster to each other.

After the maps are created, we will conduct two specific analyses: (1) a 'pattern match' that compares average cluster ratings between two groups of informants (ie, men and women, young people and stakeholders, etc) and (2) a 'Go-Zone', defined as 'a bivariate plot of the strategies values for the two rating variables used (ie, importance and feasibility) divided into quadrants above and below the mean of each rating variable'. ${ }^{30}$ The upper-right quadrant shows a 'Go-Zone' area of strategies that are above average on both rating variables in a cluster. The lowerright quadrant shows actions that have been rated above average in one variable but not the in the other and so on. This analysis will allow us to identify what strategies in each cluster need to be prioritised and/or improved on to achieve, promote and support anti-VAW masculinities.

\section{Patient and public involvement}

No patients will be involved in this study. The public will be involved as follows: at the outset of the project, two advisory groups will be established in each country, one made up of young people, and the other of stakeholders working in the arena of VAW. The advisory groups will provide input on the project design, on specific research instruments, including the interview topic guides, and on emergent findings. They will meet at pertinent points in the project (eg, in designing the interview guide, after the end of Phase 1 to discuss emergent findings, in providing feedback on the CM statements, and also interpreting findings from the CM. Please see Phase 3 for a detailed description.

\section{Phase 3: knowledge creation and translation}

The Knowledge-to-Action Framework ${ }^{32}$ is a knowledge translation model that includes 'the synthesis, dissemination, exchange and ethically sound application of knowledge to improve health'. Due to funding limitations, in this proposal, we focus on the collection of primary research (knowledge inquiry), guidelines generation (knowledge distillation) and peer-reviewed and grey literature publications (knowledge dissemination).$^{32}$

In this section, we will describe our knowledge distillation activities. These use a participatory approach where we engage with different stakeholders by involving them in the research process so that their issues and concerns are understood, considered and reflected in the project outcomes. ${ }^{33}$
Specifically, in Phase 3, and in order to engage stakeholders and young people in making sense of the findings, including the CM exercise, we will organise separate workshops with young people, community (NGOs and youth organisations) and government stakeholders (ministries of social work, health, education, gender equality, etc) who participated in Phases 1 or 2 in each country. We will also invite our advisory groups to be part of this process.

The purpose of the workshops will be to: (a) present and validate our main findings and (b) present our first draft of guidelines and materials to promote anti-VAW masculinities and challenge attitudes that tolerate VAW. We will then ask each group of stakeholders to: (1) comment on the clarity and validity of our research findings and (2) review the proposed guidelines looking at the acceptability and validity of the actions described, practical factors that may hamper their implementation, any unforeseen negative effect of the suggested strategies, costs and the current format of the content.

The feedback provided by the stakeholders will be recorded in writing and discussed within the research team. After this, we will create a document where we address all the stakeholders' comments, acknowledge any change done to the guidelines and provide justification for adopting or not the suggested changes. A final guideline document per country will be created and disseminated. Consequently, these resources will not only reflect the findings of our research, but also build on the knowledge and expertise of key stakeholders and young people.

Through stakeholders' ongoing engagement in the CM exercise and development of materials in Phase 3, we would hope to build a European network of organisations and academics concerned with addressing antiviolence masculinities, which will share approaches and interventions beyond the timescale of this project.

\section{Ethics and dissemination}

\section{Ethical clearance and informed consent}

The study was approved by the Stockholm Regional Ethical Board in Sweden (reference number 2019-02640), the human subjects research committee of Ben Gurion University in Israel (reference number 1735-1), the University College Cork Social Research Ethics Committee in Ireland (reference number 2019-053) and the ethical committee of the University of Alicante in Spain (reference number UA-2019-04-15).

WHO ethical guidelines for conducting research on VAW will be followed. Only participants aged 18-24 years will be invited to participate, which means that they can give autonomous consent. Participation will be voluntary, and the participants will be able to withdraw from the study at any time. Written informed consent from the study participants will be obtained prior to enrolment.

\section{Data protection and privacy}

Confidentiality will be protected at all times according to the European Union General Data Protection Regulation 
$(2016 / 679)$. The qualitative data collection will be conducted by the researchers in each country who have extensive experience gathering qualitative data. In-depth interviews and FGDs will be conducted in private rooms to avoid outside disturbance and ensure confidentiality. Consent will be obtained before recording the qualitative data. Quantitative data (CM phase) will be collected by means of a questionnaire.

Participants will be informed that, even though researchers are taking all precautionary measures to maintain confidentiality, complete confidentiality and anonymity cannot be guaranteed in FGDs. The researchers will remind the informants to respect the integrity of the other participants and not repeat what is said during the FGD to others. Data will be stored on university-supported secure servers. All databases will be disassociated from the participants' personal identity data before analysis.

\section{Risk assessment}

The likelihood that research participants will experience harm (psychological, social, legal, economic or environmental) is low. We will not ask participants about their own experiences of violence in close relationships, either in the interviews or FGDs, but rather about their perceptions and understandings of VAW based on specific vignettes. However, some may experience discomfort when talking about different forms of VAW or when talking about different forms of masculinities or they might spontaneously share their own experiences with VAW, which may cause distress. If this disclosure happens, and the participants appear distressed, the researcher will ask the participants if they wish to stop the interview. The researcher will also provide the participants with a list of health and support services that may be contacted in the event of distress, should they wish to speak to someone about their experience. Participants will be informed that if they are at all uncomfortable answering any questions, or do not wish to proceed, the interview can be cancelled.

If a participant experiences discomfort during a FGD, the FGD will be stopped, and he/she will be asked if he/ she wants to continue or not. Again, the participant will be offered information about appropriate support services if required.

Our dissemination strategy will target several audiences, including the academic community, policy makers, key stakeholder organisations, practitioners, educators and young people. Wherever possible, data will be made available through Open Access. Our study results will be disseminated through peer-reviewed publications, presentations at international conferences, policy briefs, social media and through the project online hub. Data will be available from the main author on request.

\section{DISCUSSION}

As discussed above, we will use a participatory, MM study combining qualitative and quantitative data in order to identify the discourses that young people and stakeholders use to conceptualise masculinities and VAW and actions needed to support and promote anti-VAW masculinities. Based on this knowledge and in cooperation with stakeholders in each country, we will develop guidelines to promote and support anti-VAW masculinities in each setting.

Masculinities are the product of gender norms and attitudes, societal structures and welfare and political systems, which coalesce within nations, but also processes that happen transnationally across borders, including new social movements; information and communications technology has been recognised as a facilitator to such transnational masculinities. Thus, a transnational perspective is vital if differences in the prevalence rates of VAW in Europe and Israel are to be understood and challenged. Adopting a transnational perspective will facilitate an exploration of how these countries' diverse sociocultural contexts-including laws, policies and welfare systemsshape young people's understandings of masculinities and VAW within and across national borders.

Studies describing the emergence of anti-VAW masculinities from violence prevention programmes have been conducted in South Africa, ${ }^{34}$ and Australia ${ }^{35}$ among other countries. These papers have provided key insights into masculinities and VAW prevention. Our current study aims to add to this knowledge by comparing how anti-VAW masculinity discourses differ and overlap across four culturally different high-income countries. In addition, our CM study will provide key data to inform the development of policies to promote and support anti-VAW masculinities in the four countries involved in this research.

\section{Author affiliations}

${ }^{1}$ Department of Global Public Health, GloSH research group, Karolinska Institutet, Stockholm, Sweden

${ }^{2}$ Department of Public Health, Faculty of Health Sciences, Ben-Gurion University of the Negev, Beer-Sheva, Israel

${ }^{3}$ School of Applied Social Studies, University College Cork, Cork, Ireland ${ }^{4}$ Institute for Social Science in the 21st Century (ISS21), University College Cork, Cork, Ireland

${ }^{5}$ Department of Community Nursing, Preventive Medicine and Public Health and History of Science, University of Alicante, Alicante, Spain

${ }^{6}$ CIBER of Epidemiology and Public Health, Madrid, Spain

Contributors MS, ND, CE, MSC and CV-C conceived the study protocol and all authors participated in its design. MS prepared a first draft of this manuscript. All authors made substantial contributions to the manuscript. All authors gave final approval for the version to be published and take public responsibility for appropriate portions of the content. All authors agreed to be accountable for all aspects of the work in ensuring that questions related to the accuracy or integrity of any part of the work are appropriately investigated and resolved. All authors read and approved the final manuscript.

Funding This work was supported by GENDER NET Plus Co-Fund (reference number 2018-00968).

Disclaimer The funders will have no role in study design, data collection, analyses, publishing or manuscript writing.

Competing interests None declared.

Patient and public involvement Patients and/or the public were involved in the design, or conduct, or reporting, or dissemination plans of this research. Refer to the Methods section for further details.

Patient consent for publication Not required. 
Provenance and peer review Not commissioned; externally peer reviewed.

Open access This is an open access article distributed in accordance with the Creative Commons Attribution Non Commercial (CC BY-NC 4.0) license, which permits others to distribute, remix, adapt, build upon this work non-commercially, and license their derivative works on different terms, provided the original work is properly cited, appropriate credit is given, any changes made indicated, and the use is non-commercial. See: http://creativecommons.org/licenses/by-nc/4.0/.

ORCID iD

M Salazar http://orcid.org/0000-0001-6935-9781

\section{REFERENCES}

1 United Nations. Declaration on the elimination of violence against women. Geneva: United Nations, 1994.

2 FRA - European Union Agency for Fundamental Rights. Violence against women: an EU-wide survey. main results. Luxembourg: Publications Office of the European Union, 2015.

3 Daoud N, Sergienko R, Shoham-Vardi I. Intimate partner violence prevalence, recurrence, types, and risk factors among Arab, and Jewish immigrant and Nonimmigrant women of childbearing age in Israel. J Interpers Violence 2020;35:2869-96.

4 Conell RW. Masculinities. 2nd edn. Los Angeles,CA: University of California Press, 2005

5 Jewkes R, Sikweyiya Y, Morrell R, et al. Gender inequitable masculinity and sexual entitlement in rape perpetration South Africa: findings of a cross-sectional study. PLoS One 2011;6:e29590.

6 Barker G, Contreras JM, Heilman B, et al. Evolving men. initial results from the International men and gender equality survey (IMAGES). Washington, DC: International Center for Research on Women (ICRW) and Instituto Promundo, 2011.

7 Fulu E, Jewkes R, Roselli T, et al. Prevalence of and factors associated with male perpetration of intimate partner violence: findings from the UN Multi-country cross-sectional study on men and violence in Asia and the Pacific. Lancet Glob Health 2013;1:e187-207.

8 Salazar M, Öhman A. Negotiating Masculinity, Violence, and Responsibility: A Situational Analysis of Young Nicaraguan Men's Discourses on Intimate Partner and Sexual Violence. J Aggress Maltreat Trauma 2015;24:131-49.

9 Salazar Torres VM, Goicolea I, Edin K, et al. 'Expanding your mind': the process of constructing gender-equitable masculinities in young Nicaraguan men participating in reproductive health or gender training programs. Glob Health Action 2012;5.

10 Goicolea I, Torres MS, Edin K, et al. When sex is hardly about mutual Pleasure: dominant and resistant discourses on sexuality and its consequences for young people's sexual health. Int J Sex Health 2012;24:303-17.

11 Connell RW, Messerschmidt JW. Hegemonic Masculinity:Rethinking the Concept. Gender Society 2005;19:829-59.

12 Allen A. Feminist Perspectives on Power. In: Zalta EN, ed. The Stanford encyclopedia of philosophy, 2016. https://plato.stanford. edu/archives/fall2016/entries/feminist-power/

13 Connell RW. Teaching the boys: new research on masculinity, and gender strategies for schools. Teach Coll Rec 1996;98:206-35.

14 Howson R. Challenging hegemonic masculinity. London; UK: Routledge, 2006.

15 Elliott K. Caring masculinities: theorizing an emerging concept. Men Masc 2016;19:240-59.
16 Flood M. Men's antiviolence activism and the construction of genderequitable masculinities. In: Carabi A, Armengol J, eds. Alternative masculinities for a changing world. New York: Palgrave Macmillan, 2015: 35-50.

17 Glick P, Fiske ST, Mladinic A, et al. Beyond prejudice as simple antipathy: hostile and Benevolent Sexism across cultures. J Pers Soc Psychol 2000;79:763-75.

18 Jewkes R, Flood M, Lang J. From work with men and boys to changes of social norms and reduction of inequities in gender relations: a conceptual shift in prevention of violence against women and girls. Lancet 2015;385:1580-9.

19 European Commission. Strategy for equality between women and men 2010-2015. Luxembourg: Publications Office of the European Union, 2010.

20 Andersson K. Constructing young masculinity: a case study of heroic discourse on violence. Discourse \& Society 2008;19:139-61.

21 Valls R, Puigvert L, Duque E. Gender violence among teenagers: socialization and prevention. Violence Against Women 2008;14:759-85.

22 Marcos Marcos J, Avilés NR, del Río Lozano M, et al. Performing masculinity, influencing health: a qualitative mixed-methods study of young Spanish men. Glob Health Action 2013;6:21134.

23 Hearn J. Hegemonic masculinity and beyond: 40 years of research in Sweden. Men Masc 2012;15:31-55.

24 Stöckl H, March L, Pallitto C, et al. Intimate partner violence among adolescents and young women: prevalence and associated factors in nine countries: a cross-sectional study. BMC Public Health 2014;14:751.

25 Curry LA, Krumholz HM, O'Cathain A, et al. Mixed methods in biomedical and health services research. Circ Cardiovasc Qual Outcomes 2013;6:119-23.

26 Palinkas LA, Horwitz SM, Green CA, et al. Purposeful sampling for qualitative data collection and analysis in mixed method implementation research. Adm Policy Ment Health 2015;42:533-44.

27 Clarke A. Situational analysis:Grounded theory after the postmodern turn. Thousand Oaks, CA: Sage publications, 2005.

28 Wetherell M, Potter J. Mapping the language of racism:Discourse and the legitimation of exploitation. London and New York: Harvester Wheatsheaf and Columbia University Press, 1992.

29 Graneheim UH, Lundman B. Qualitative content analysis in nursing research: concepts, procedures and measures to achieve trustworthiness. Nurse Educ Today 2004;24:105-12.

30 Kane M, Trochim WM. Chapter 1. An Introduction to Concept Mapping. In: Concept mapping for planning and evaluation. Thousand Oaks: SAGE Publications, 2007.

31 Hout MC, Papesh MH, Goldinger SD. Multidimensional scaling. Wiley Interdiscip Rev Cogn Sci 2013;4:93-103.

32 Straus SE, Tetroe J, Graham I. Defining knowledge translation. CMAJ 2009;181:165-8.

33 Department of Health. Stakeholder engagement framework. Canberra: Australian Goverment, 2017. https://www.health.gov.au/ resources/publications/stakeholder-engagement-framework

34 Dworkin SL, Hatcher AM, Colvin C, et al. Impact of a GenderTransformative HIV and Antiviolence program on gender Ideologies and masculinities in two rural, South African communities. Men Masc 2013;16:181-202.

35 Bell K, Flood M. Chapter 4. Change among the Change Agents? Men's Experiences of Engaging in Anti-Violence Advocacy as White Ribbon Australia Ambassadors. In: Luyt R, Starck K, eds. Masculine power and gender equality: masculinities as change agents. Generva, Switzerland: Springer Nature Switzerland AG, 2020. 